\title{
Motivate: towards Context-Aware Recommendation Mobile System for Healthy Living
}

\author{
Yuzhong Lin, Joran Jessurun, Bauke de Vries, Harry Timmermans \\ Eindhoven University of Technology \\ Eindhoven, the Netherlands
}

\begin{abstract}
This paper presents the practices of a research aiming at the design of a context-aware recommendation system that promotes the adoption of a healthy and active lifestyle. A Smartphone application that provides personalized and contextualized advice on physical activities was developed. The goal of our endeavor is to find out whether this recommendation system can generate motivating advice at an appropriate time and location by taking environment, weather, user location and agenda into account. A small evaluation user study was conducted and the results show the feasibility of a real-time context-aware recommendation system on Smartphone.
\end{abstract}

Keywords-wellness; physical activity; context-aware; mobile application; design; user study.

\section{INTRODUCTION}

According to WHO by 2015 , approximately 2.3 billion adults will be overweight and more than 700 million will be obese [1]. Overweight and obesity can lead to many serious health problems. It is a challenge to stay motivated to maintain a sufficient amount of physical activity. Nowadays with the development of mobile technologies, mobile device may act as powerful persuaders because they can intervene in the right context and a convenient way in order to prompt users into behavior change [2]. The mobile device tracks context and prompts users to take action, as Fogg's description of one persuasive role of mobile phones - "coach" [3]. For example, a project developed by Kass [4] aspired to transform a Smartphone into a virtual coach to provide users with continuous, automated coaching based on their physiological data. Another application called BeWell Mobile system transmitted personalized messages to patients' mobile phones to control various diseases by triggering behavior change [5].

Nowadays, ubiquitous computing and context-aware persuasive technologies [2] offer a new healthcare opportunity to promote health behavior by presenting "just-in-time information." [6] [7]. Intille [7] suggested an effective strategy of presenting just-in-time information at an appropriate time and an appropriate place to motivate behavioral change. The main focus of our system design is to motivate people to behave more physically active by providing recommending messages on their mobile device at an appropriate time and place. To give advice at the right location the system requires information of user location and the surrounding environment, while to give advice at the right moment the system requires knowledge about what users' activities are. The advice on physical activity must fit easily into users' daily routine. The reason is that messages suggesting simple activities are preferred over ones requiring significant effort [8]. Besides, lifestyle interventions can yield positive and long-term effects, in terms of increasing the levels of moderately intense physical activity [9]. The suggested locations of lifestyle activities we include in our system are [9]: everyday activity destinations (shops, homes, schools, workplaces etc.) and informal activity and recreation (playgrounds, parks and gardens etc).

The newest trend of using Smartphone and location-based technologies makes the just-in-time information presented at a right location and time feasible. The findings of a recent survey of the usage of healthcare applications on Smarphone showed an optimistic future for mobile health and wellness applications [10]. Bearing this in mind we started off with the concept design of a context-aware recommendation system that requests user location, environment, weather, user agenda and profile as the input context. To prove the acceptance of this concept design, we first did a small scale user study [11] and a technical simulation [12]. Afterwards we carried on with further development of a real-time mobile application.

In this paper, we present a Smartphone application "Motivate" that provides users with personalized and contextualized advice on possible physical activities to do. We introduce the design, implementation and an evaluation test of the "Motivate" system.

\section{IMPLEMENTATION}

This section describes the implementation of "Motivate" software system which is built on Ruby on Rails framework.

\section{A. System architecture}

Motivate system consists of Motivate service, Motivate web application and Mobile API which communicates with Motivate mobile application. The "Motivate" application is compatible with Android phone version 2.0 and above. The application sends the phone location detected by either GPS or GSM localization. If there is suitable advice generated by the Motivate Service, it is sent to users' mobile phone. Then users can send back their responses to the Motivate Server. The Motivate web application is implemented for users to edit their profile and personal agenda. The administrator can also use this web application to easily manage user data and observe how users are using the mobile application. The system architecture is illustrated in Fig. 1. 


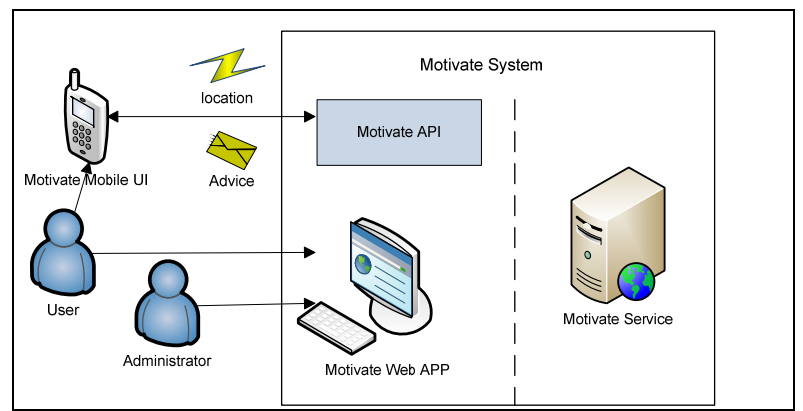

Figure 1. System Architecture

\section{B. Motivate Service}

The Motivate Service consists of services including Advisor, Location, Agenda, Weather, Profile and Time. After receiving a request containing user location, the Advisor

Service tries to find a proper advice from the advice database by asking the other services. If any good advice is found, the Advisor Service creates a message to send to the mobile UI and then store it in the Message Database. Fig. 2 shows the Motivate Service with the services and database.

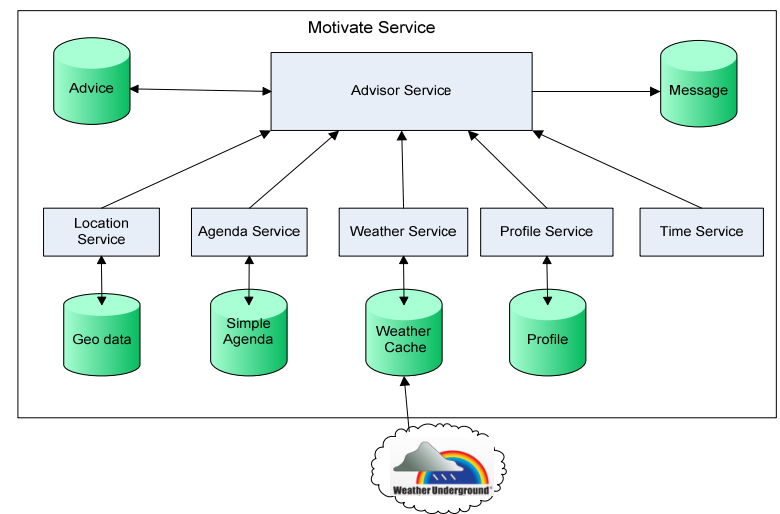

Figure 2. Motivate Service

\section{1) Location Service}

We use PostGIS to manage the geo database. The geo database is created by adding geographic information of green places, such as parks, lakes, forests provided by OpenStreetMap [13]. Shopping centers, markets, cinemas, and some museum were added to the database as well. In addition the users' significant locations, namely home and work place were implemented in the geo database. This can be done by using the Motivate web application by administrator. When the Location Service receives a query from the Advisor, it analyzes the user location by calculating the distance in meters projected on a local spatial reference system. The location analysis shows the position of the user with respect to other locations present in database.

\section{2) Agenda Service}

We developed a Simple Agenda Service on our Motivate web application. Users can add activities from a drop-down list which contains: "Go to work", "Work", "Lunch", "Go home", "Dinner" and "Busy". They can specify the starting and ending time of that activity. It is a weekly schedule and users can copy the agenda of another day in the week to easily set up the whole week agenda. The Simple Agenda deals with the query by checking what the user's current activities or future activities are within 3 hours are as indicated in the agenda. Only the entry called "Busy" indicates the user does not want any advice for that period.

\section{3) Weather Service}

The weather history data is retrieved from the website of Weather Underground [14] and stored in whether cache. The weather information is recorded every 20 minutes on average. The information contains time, and weather conditions. Certain criteria are adopted for labeling weather state as bad (e.g., rainy or temperature lower than $5^{\circ} \mathrm{C}$, fair (e.g., cloudy and temperature higher than $5^{\circ} \mathrm{C}$ ) or good (e.g., sunny and temperature $15^{\circ} \mathrm{C} \sim 25^{\circ} \mathrm{C}$ ) based on the data from the website. The Weather Service updates the database and picks up the weather record with the time closest to the query time.

\section{4) Profile Service}

Users edit their profile such as gender, age, family status, transportation, home, work place address, etc by using the Motivate web application.

\section{5) Advisor}

The Advisor service calculates for each advice in the advice database its suitability for the given situation. Each advice consists of a process and a template. The If-Then rules defined in the "process" specify which constraints must be met for each advice. The Advisor Service sends a query to one or more of the Services and acquires their analysis results. If all the constraints are met, the advice becomes a candidate. If more than one piece of advice is activated, one randomized advice is chosen. We keep track of the sent messages and make sure users do not receive the same or similar kind of advice repeatedly.

There were in total 34 pieces of advice including 20 kinds of activities with different constraints. The constraints are :

- Location: suggested activity location must be within a certain distance or travel time.

- $\quad$ Agenda: suggested activity should be sent when a user goes to work, has dinner, etc. Users should have a free agenda to conduct this activity.

- Weather: outdoor activity advice requires good or fair weather conditions.

- Profile: advice such as cycling to work requires users' possession of bike.

- Time: suggested activity is applicable for a certain specified time period (e.g., Saturday morning to go to the market)

One example of a "Lunch walk advice" contains the following constrains: the day of week is a weekday; a user's agenda activity "Lunch" starts within twenty minutes; a user's agenda is free for one hour; the current weather is fair; there is a green place within walking time (within $300 \mathrm{~m}$ for a short walk). The field "template" of the chosen advice can dynamically compile the text of the message to be sent. In this example the template is "It's pretty good weather outside, how 
about a short walk to $<$ the name of suggested the green place in the geo database $>$ during lunch break?"

\section{Motivate Mobile User Interface}

The Android phone application serves as location detector and user interface that interacts with users. The application is developed using the Android Software Development Kit. On the application main page, users can see the total number of advice they have received and the frequency of replying positively or negatively to the messages (see Fig. 3, left). Users can set "preferences" for the time interval between times of checking location, notification sound and other basic setting. The "Edit agenda" button is to direct users to the Simple Agenda page of Motivate web application.

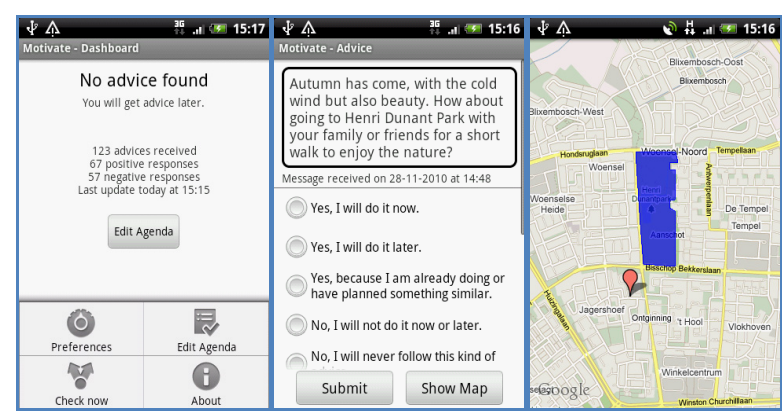

Figure 3 Motivate mobile APP interface

If there is a message found, a notification is sent to a user. A message (see Fig. 3, middle) is shown on the screen and there are choices of responses for the user to choose. The 5 possible responses are as follows:

- "Yes, I will do it now"

- "Yes, I will do it later"

- $\quad$ "Yes, because I am already doing or have planned something similar"

- "No, I will not do it now or later"

- "No, I will never follow this kind of advice"

After users give their response they can also type their reason for their choice. Afterwards they are asked about if the timing of the message was "just in time", "too late" or "too early". By pressing the "Show Map" button, a user can view the suggested place on Google map and where he or she is at that moment (see Fig. 3, right). The questions are designed in order to collect user feedback to each advice for evaluation. We are interested in user's intention to follow the advice and how they perceive the timing of the advice.

\section{EVALUATION TEST SETTING}

In order to evaluation the "Motivate" system we conducted a user study starting from 15 November to 25 December, 2010. Participants of the user test consisted of 6 Android phone users (5 male, 1 female). The average age of participants was 37 years (range: 24-63 years). They worked 5 days a week. According to Body mass index (BMI) measurement, 5 of them are with normal weight and 1 of them is slightly overweight. Due to the limited number of participants required in this phase we only contacted possible participants which were easy to approach (e.g. colleagues, friends)

Two participants were using HTC Hero mobile phones and the other four with Sumsung GalaxyS. Participants were invited for a set-up meeting. They download the "Motivate" Android application on their mobile phone. The default setting checked every 15 minutes for advice. Participants were instructed edit their personal profile and agenda. For the first week participants did not get any advice but their coordinates were sent to the Motivate Service. The data including date, time latitude and longitude was saved as a KML file to be shown on Google Earth (see Fig.4). From the tracks we collected in the assessment period, we discovered a certain movement pattern, namely two points of most frequent locations, which were home and work place of a participant. We verified this with their self-reported home and work address and added those places into each participant's geo database (see Fig.4). After one week of assessment, messages of physical activity advice were sent to participants' mobile phone through the "Motivate" application. They were instructed to give their response to each message for evolution purpose. After 5 weeks participants were invited for a face-toface interview about their user experience.

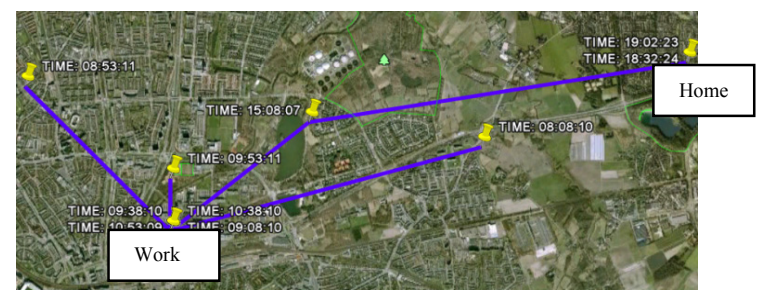

Figure 4. Location points of one participant for one day

\section{RESULTS \& DISCUSSIONS}

\section{A. System performance}

During these 5 weeks, 464 messages were sent in total for all participants. On average 3 messages were sent for each participant per day. The percentages of messages sent in the morning, afternoon and evening were $45.3 \%$, 25\% and $29.6 \%$ respectively. We categorized messages into 5 types. The percentage of different message types is shown in Table I. The messages of work, home and outdoor category were sent more often compared to other messages. The most frequently sent message suggested taking a break during work (14.5\%) and taking the stair when arriving or leaving work (10.5\%).

TABLE I MESSAGES OF 5 TYPES

\begin{tabular}{|c|c|c|}
\hline Types & Examples & $\begin{array}{c}\text { Percentage } \\
\text { of messages }\end{array}$ \\
\hline Work & Take a break, take stairs & $30.0 \%$ \\
\hline Home & Housework, stretching, gardening & $24.9 \%$ \\
\hline Outdoor & Walk/cycling to a park & $24.3 \%$ \\
\hline Indoor & Shopping, go to museum or exhibition & $13.1 \%$ \\
\hline Transportation & Cycle/walk to work, take a detour home & $7.7 \%$ \\
\hline
\end{tabular}




\section{B. User feedback to messages}

Out of 464 messages, 434 responses were given by the users. In total $50.5 \%$ of the messages received positive responses which includes "Yes-Now", "Yes-Later" and "YesAlready", $47 \%$ of the messages were given negative response of "No" and 2.5\% "Never". Almost $75 \%$ of the messages about activity that at work received positive responses, which was the highest percentage compared to other categories. Among all kinds of advice we noticed that the easy-to-do activities such as "coffee break" or "taking the stairs" were sent to users more often and those messages received more positive responses as well. Although these small activities do not increase the physical activity to a level of calorie burning, they do help users to adopt a healthy lifestyle by changing habits. This is in line with the reason that we only include simple daily activities in the advice database [9]. 57.1\% of all the messages were considered to be sent just in time, while $25.6 \%$ of them were too late and $17.3 \%$ of them were too early Half of messages were considered as just in time, which indicates requirements for future improvements.

We analyzed the reason given by participants in the cases that they gave a negative response. A number of 106 out of 215 negative responses were given an explanation by participants, underlying their reason of not following the advice. The reason mentioned most were "busy at the moment, have no time" (24.5\%), "already have other plans" (19.8\%) and "feel no need to do the activities" (17\%). Other reasons were "bad weather", "I feel tired", "It's not feasible" and "I don't feel like it".

\section{User Interview}

The advice participants liked most were daily activities that they tend to forget but are easy to do such as walking to the coffee corner for a break, or a short lunch walk. Although we implemented "Agenda" in order to prevent sending advice when they already had scheduled activities, it did not work out as we expected. In fact participants reported that they did not update their agenda by adding the "busy" time slot, especially during the weekend. Thus the function of this simple agenda is mainly indicating the rough schedule of basic activities to determine the best timing. For the nearby future new solutions will be proposed to enhance the usage of the "Agenda". One possible solution is to send the advice in advance in order to give users more time to prepare. Users should be able to indicate when they plan to follow the advice and get a reminder later as well.

\section{CONCLUSIONS}

In this paper we describe the design, implementation and evaluation user test of a mobile application "Motivate". The results show us the applicability of a real-time context-aware recommendation system on Smartphone. With the state of the art technology we made personalized and contextualized recommendation available for the user's mobile device at any time. The location, agenda and weather input is simple but sufficient for analyzing user context. The if-then-rules that take multiple inputs into account to generate different kinds of advice were proven to be satisfactory. The application can be beneficial for people who want to pursue a healthy lifestyle, especially for those who have a regular job and participate insufficient in physical activity. The "Motivate" can be a gentle push and reminder for simple daily activities, as well as offering innovative activity ideas for their free time

This evaluation test focused mainly on the usage of the application and possible reactions from users. Since in this user study we did not take any measurement of the physical activities of the participants, we can hardly conclude whether they became more active during this period. As future work the timing of the advice needs further improvements. Besides we will improve the user interface with more interactive questions in order to learn from user preference and make the advice more personalized. The next step is a bigger scale experiment involving more participants. This experiment is scheduled for this spring and its results will give us a better insight into the usage of mobile device in the field of behavioral change.

\section{REFERENCES}

[1] WHO Media centre, Obesity and overweight, Sep. 2006. [online] Avalibale: http://www.who.int/mediacentre/factsheets/fs311/en/. [Accessed: Feb. 10, 2011]

[2] B. J. Fogg, Persuasive Technology: Using Computers to Change What We Think and do. San Francisco, CA: Morgan Kaufmann, 2003.

[3] B. J. Fogg and D. Eckles, Ed., Mobile Persuasion: 20 Perspective on the Future of Behavior Change. California: Stanford Captology Media, 2007.

[4] A. Kass, "Transforming the mobile phone into a personal performance coach," in Mobile Perusasion: 20 Perspective on the Future of Behavior Change, Under Development, B. J. Fogg, and D. Eckles, Ed. California: Stanford Captology Media, 2007, pp. 39-45.

[5] P. Boland, "Managing chronic diserse through Mobile Persuasion," in Mobile Perusasion: 20 Perspective on the Future of Behavior Change, Early Movers, B. J. fogg, and D. Eckles, Ed. California: Stanford Captology Media, 2007, pp. 39-45.

[6] S. S. Intille, "A new research challenge: Persuasive technology to motivate healthy aging," IEEE Transactions on Information Technology in Biomedicine, Vol.8, pp. 235-237, Sep. 2004.

[7] S. S. Intille, "Ubiquitous Computing Technology for Just-in-Time Motivation of Behavior Chang," in MedInfo 2004, M. Fieschi, E. Coiera and Y. Li, Ed. Amsterdam: ISO Press, 2004 , pp. 1434-1437.

[8] M. Maheshwari, S. Chatterjee and D. Drew. "Exploring the persuasiveness of "Just-in-time" motivational messages for obesity management", In: Oinas-Kukkonen, H. et al. PERSUASIVE 2008, LNCS 4022, pp. 258-261. Springer, Heidelberg ,2008.

[9] L. Frank, P. Engelke and T. Schimid. Health and Community Design: the Impact of the Built Environment on Physical Activity. Island Press, Washington, DC , 2003.

[10] Euro RSCG Life 4D, "The promis of mobile health", November, 2010. Available:http://www.eurorscglife.com/sites/default/files/u12/4THOUG HT.pdf [Available, Feb.10. 2011]

[11] Y. Lin, B. de Vries and H.J.P. Timmermans. "Design of a ContextAware Mobile Persuasive System for the promotion of a healthier lifestyle." In: Proceedings of Poster Papers for the Fifth International conference on Persuasive Technology, pp.85-88. 2010.

[12] Y. Lin, A. J. Jessurun,B. de Vries and Timmermans, H.J.P. "A contextaware persuasive system for active living -simulator system design." In: Conference proceeding of 10th International Conference on Design \& Decision Support Systems in Architecture and Urban Planning, pp.1-12. 2010.

[13] OpenStreetMap data, [online] Available : http://download.geofabrik.de/osm/europe/ [Accessed: Feb. 10, 2011]

[14] Weather undergrond, http://www.wunderground.com/ 